Agrotrópica 32(3): 217 - 224. 2020.

Centro de Pesquisas do Cacau, Ilhéus, Bahia, Brasil

\title{
DESENVOLVIMENTO DE PLANTAS DE Eucalyptus urocam SOB DÉFICIT HÍDRICO E DIFERENTES REGULADORES VEGETAIS
}

\author{
Víctor Alves Amorim, Brunno Nunes Furtado, Felippe Baptista de Alencastro, Igor Alberto \\ Silvestre Freitas, Winy Kelly Lima Pires, Larissa Pacheco Borges, Fábio Santos Matos \\ Universidade Estadual de Goiás, Grupo de Pesquisa da Fisiologia da Produção Vegetal. Rodovia GO 330 km 241, Anel \\ Viário, 75780-000, Ipameri, Brasil. victor.alves.a@gmail.com; fabio.agronomia@hotmail.com
}

\begin{abstract}
Objetivou-se identificar os efeitos de reguladores vegetais no desenvolvimento de plantas de Eucalyptus urocam e os mecanismos de tolerância a deficiência hídrica da espécie. O experimento foi conduzido em casa de vegetação seguindo o delineamento inteiramente casualizado em esquema fatorial $2 \times 5$ (hormônio x níveis de irrigação) com cinco repetições. As mudas de E. urocam foram submetidas aos níveis de irrigação de 40, 60, 80, 100 e $120 \%$ da evapotranspiração diária. Aos 15 dias após transplantio foi feita a primeira aplicação da mistura (brassinosteroides, cinetina e giberelina nas concentrações $0,1 \mathrm{mg} \mathrm{L}^{-1}, 2 \mathrm{mg} \mathrm{L}^{-1} \mathrm{e} 2 \mathrm{mg} \mathrm{L}^{-1}$ respectivamente) totalizando quatro aplicações. Aos 10 dias após a última aplicação foram analisadas as variáveis de crescimento, pigmentos fotossintéticos, teor relativo de água, transpiração e parâmetros de fluorescencia. A aplicação dos reguladores não apresentou efeito significativo no crescimento das plantas, no entanto, os diferentes volumes de água interferiram de forma linear na biomassa, área foliar, transpiração e máxima eficiência quântica do FSII. No entanto o uso de reguladores vegetais em mudas de E. urocam não promove variações no desenvolvimento destas plantas quando submetidas a diferentes níveis de irrigação, por isso, não se justifica o uso em mudas desta espécie.
\end{abstract}

Palavras-chave: hormônio, silvicultura, seca, mudas florestais.

Development of Eucalyptus Urocam plants under water deficit and different plant regulators. The objective was to identify the effects of plant regulators on the development of Eucalyptus urocam plants and the mechanisms of tolerance to water deficiency of the species. The experiment was conducted in a greenhouse following a completely randomized design in a $2 \times 5$ factorial scheme (hormone $\mathrm{x}$ irrigation levels) with five replications. E. urocam seedlings were subjected to irrigation levels of 40, 60, 80, 100 and $120 \%$ of daily evapotranspiration. At 15 days after transplantation, the first application of the mixture (brassinosteroids, kinetin and gibberellin in concentrations $0.1 \mathrm{mg} \mathrm{L}^{-1}, 2 \mathrm{mg} \mathrm{L}^{-1}$ and $2 \mathrm{mg} \mathrm{L}^{-1}$ respectively) was made, totaling 4 applications. At 10 days after the last application, growth variables, photosynthetic pigments, relative water content, transpiration and fluorescence parameters were analyzed. The application of the regulators did not have a significant effect on the growth of the plants, however, the different volumes of water interfered in a linear way in the biomass, leaf area, transpiration and maximum quantum efficiency of the FSII. However, the use of plant regulators in E. urocam seedlings does not promote variations in the development of these plants when submitted to different levels of irrigation, therefore, the use in seedlings of this species is not justified.

Key words: hormone, forestry, drought, forest seedlings. 


\section{Introdução}

A utilização irracional da água e a intensificação do uso de fontes energéticas poluidoras são atividades antrópicas que levam a degradação do meio ambiente (Glantz, 2019). Para prevenir as futuras gerações de crises na agricultura é necessário desenvolver tecnologias e manejos que minimizem os efeitos do déficit hídrico através do uso eficiente da água e desenvolvimento de plantas tolerantes (Maggio et al., 2018).

A demanda de madeira no mundo aumenta constantemente tornando-a muito importante na matriz energética de países em desenvolvimento como o Brasil em que a produção e utilização são crescentes (Antwi-Boasiako \& Acheampong, 2016). Segundo a Food and Agriculture Organization - FAO (2018) estima-se que a madeira fornece mais da metade da cadeia primária energética em 29 países, sendo 22 destes na África.

A cadeia de produção do setor florestal gera níveis substanciais de emprego. Mais de 13 milhões de pessoas estão formalmente empregadas em atividades do setor florestal (Arce, 2019). Na esfera informal de pequenas e médias empresas florestais (PMEs), outras 40 a 60 milhões de pessoas podem ser empregadas e, em alguns países, as PMEs tem 80 a $90 \%$ do emprego relacionado à floresta (Arce, 2019). O cultivo de espécies florestais desempenha papel fundamental no crescimento econômico de forma sustentável, principalmente em pequenas propriedades (Jong et al., 2018).

As espécies do gênero Eucalyptus são muito utilizadas no cultivo florestal no mundo, consolidandose como importante matéria prima para diferentes indústrias (Mcmahon \& Jackson, 2019). A madeira do eucalipto pode ser utilizada em compensados, pavimentos, serraria, mobília, papel, celulose, carvão, biomassa, entre outros (França et al., 2019). O gênero abrange mais de 960 espécies e apresentam diferenças genotípicas adaptadas para diferentes ambientes ocupando regiões como a Ásia, Europa, África e América do Sul (Brooker, 2000). Apesar do sucesso das florestas plantadas, o setor pode gerar ainda mais riqueza se explorar áreas impróprias para várias outras culturas menos tolerantes ou sensíveis a estresses abióticos (Lopes et al., 2015).
Os estresses abióticos incluem fatores como salinidade, seca e altas temperaturas que causam reduções no crescimento das plantas no mundo (Dresselhaus \& Hückelhoven, 2018). As plantas respondem de várias formas aos estresses e os hormônios vegetais fazem parte de uma interação complexa nessas respostas (Awan, Khurshid \& Mehmood, 2017). É necessário utilizar uma combinação de recursos biotecnológicos para as plantas poderem expressar o potencial máximo em situações desfavoráveis ao crescimento (Maggio et al., 2018).

Estudos mostram serem necessários a identificação de estratégias de tolerância a escassez de água que facilitem o estabelecimento e posterior crescimento inicial do vegetal e, além disso, implantar alternativas promissoras de manejo como o uso de hormônios vegetais e elicitores que ativam mecanismo de tolerância a seca.

Os reguladores vegetais são mensageiros químicos de sinalização que em baixa concentração exerce ação estimulante no metabolismo das plantas. O uso de hormônios representa importante alternativa de manejo de espécies vegetais no intuito de obter incrementos no crescimento e/ou ativação de mecanismos de tolerância a estresses abióticos (elicitores). Sendo assim, o presente estudo teve como objetivo identificar os efeitos de reguladores vegetais no desenvolvimento de plantas de Eucalyptus urocam e os mecanismos de tolerância a deficiência hídrica da espécie.

\section{Material e Métodos}

O experimento foi conduzido sobre bancada da casa de vegetação na Universidade Estadual de Goiás, Câmpus Ipameri (Lat. $17^{\circ} 43^{\prime}$ 19” S, Long. 48 09' 35 " W, Alt. 773 m), Ipameri, Goiás. De acordo com a classificação de Köppen o clima é do tipo tropical chuvoso (Aw), com temperatura média do mês mais frio maior que $18{ }^{\circ} \mathrm{C}$, com inverno seco e chuvas máximas no verão.

As mudas de E. urocam com 100 dias de idade foram transplantadas em vasos de cinco litros com 5,5 $\mathrm{kg}$ de substrato contendo uma mistura de solo, areia e esterco na proporção de $3: 1: 0,5$, respectivamente. A análise química da mistura revelou os seguintes valores: $\mathrm{pH}\left(\mathrm{CaCl}_{2}\right) 5.4 ; 16 \mathrm{~g} \mathrm{dm}^{-3}$ de matéria orgânica; $68 \mathrm{mg}$ $\mathrm{dm}^{-3}$ de P; 6.81 mmolc dm $^{-3}$ de K $\left(\right.$ Mehlich $\left.^{-1}\right) ; 22$ 
mmolc dm ${ }^{-3}$ (Tampão SMP) de $\mathrm{H}+\mathrm{Al} ; 31$ mmolc dm ${ }^{3} \mathrm{de} \mathrm{Ca} ; 15$ mmolc dm ${ }^{-3} \mathrm{de} \mathrm{Mg} ; 53$ mmolc $\mathrm{dm}^{-3} \mathrm{de} \mathrm{SB}$; 75 mmolc dm ${ }^{-3}$ de CTC; e $71 \%$ de saturação por bases. Após interpretação da análise da mistura, decidiu-se não realizar calagem e adubação.

As mudas foram submetidas a diferentes níveis de irrigação de 40, 60, 80, 100 e 120\% da evapotranspiração diária. Como o coeficiente da cultura $(\mathrm{kc})$ para eucalipto ainda não foi determinado na região de Ipameri, GO, utilizamos o kc igual a 1,00 seguindo estimativa da FAO 56 (Allen et al., 1998) para um grupo de culturas em estádio de crescimento inicial.

O volume de água fornecido foi estimado determinando a evapotranspiração de referência e o coeficiente da cultura. Para determinar a evapotranspiração da cultura, utilizou-se a equação:

$\mathrm{ETc}=\mathrm{ETo} \mathrm{x} \mathrm{kc}$

Onde:

$\mathrm{ETc}=$ evapotranspiração da cultura

$\mathrm{kc}=$ coeficiente da cultura

ETo $=$ Evapotranspiração de referência

O cálculo da ETo diária foi feito pelo método de Penman-Monteith recomendado pela FAO (Smith, 1991) utilizando os dados diários de temperatura máxima e mínima do ar, umidade relativa do ar, insolação e velocidade do vento obtidos na Estação Meteorológica do INMET localizado no município de Ipameri, GO.

Aos 15 dias após transplantio das plantas dos tubetes para os vasos foi feita a primeira aplicação da mistura de hormônios (brassinosteroides, cinetina e giberelina nas concentrações $0,1 \mathrm{mg} \mathrm{L}^{-1}, 2 \mathrm{mg} \mathrm{L}^{-1} \mathrm{e} 2$ $\mathrm{mg} \mathrm{L}^{-1}$ respectivamente) em volume de $10 \mathrm{ml} /$ planta/ aplicação em intervalos de 10 dias, totalizando 4 aplicações. As aplicações ocorreram sempre no início da manhã entre 7:30 e 8:00 h.

Aos 10 dias após a última aplicação foram analisadas as seguintes variáveis: altura de planta, diâmetro de caule, área foliar, biomassa, razão de massa radicular (RMR), razão de massa caulinar (RMC), razão de massa foliar (RMF), transpiração, teor relativo de água, coeficiente de extinção fotoquímica (qP), coeficiente de extinção não fotoquímica (NPQ), fluorescência inicial $\left(\mathrm{F}_{\mathrm{o}}\right)$, fluorescência máxima $(\mathrm{Fm})$, rendimento quântico máximo do fotossistema II ( $\mathrm{Fv} / \mathrm{Fm})$, eficiência de captura de energia de excitação (Fv'/Fm') e energia absorvida não direcionada a fotoquímica nem dissipada por calor $(\mathrm{Pe})$ e concentrações foliares de carotenoides e clorofilas totais.

Variáveis de crescimento: A altura de planta foi mensurada a partir da região de transição da raiz com o caule na base da planta rente ao solo (coleto) até o ápice do caule utilizando régua milimétrica. $\mathrm{O}$ diâmetro do caule foi mensurado na altura do coleto com paquímetro digital. O número de folhas foi obtido por contagem. As folhas, raízes e caules foram destacados e colocados para secar em estufa a $72{ }^{\circ} \mathrm{C}$ por $72 \mathrm{~h}$ até atingir massa seca constante e, em seguida, pesados separadamente. Com os dados de massa seca calculou-se a biomassa total somando-se as massas de todas as partes da planta e razões de massa foliar, caulinar e radicular dividindo-se a massa do órgão específico pela biomassa total.

Pigmentos fotossintéticos: Para a determinação da concentração de clorofilas e carotenoides totais, foram retirados dois discos foliares de $0,6 \mathrm{~mm}$ de diâmetro cada, em folhas totalmente expandidas e colocados em vidros contendo $3 \mathrm{ml}$ de dimetilsulfóxido (DMSO) saturado com carbonato de cálcio $\left(50 \mathrm{~g} \mathrm{~L}^{-1}\right)$. Posteriormente, realizou-se a extração em banho-maria a $65^{\circ} \mathrm{C}$ por uma hora. Retirou-se alíquotas para leitura espectrofotométrica a 480, 646 e $663 \mathrm{~nm}$. O conteúdo de clorofila a $(\mathrm{Cl} a)$, clorofila b $(\mathrm{Cl} b)$ e carotenoides totais (Car) foram determinados seguindo a equação proposta por Wellburn, (1994).

Teor relativo de água na folha: Para obtenção do teor relativo de água, foram retirados cinco discos foliares de 1,2 cm de diâmetro em folhas totalmente expandidas, pesados para registrar a massa fresca (MF) e colocados para saturar por 24 horas em placas de petri com água destilada quando foram novamente pesados e em seguida determinada a massa túrgida (MT), posteriormente foram colocados para secar à temperatura de $70^{\circ} \mathrm{C}$ por 72 horas, sendo então obtida a massa seca (MS) e, em seguida foi calculado o teor relativo de água seguindo a equação:

$$
(\mathrm{MF}-\mathrm{MS}) /(\mathrm{MT}-\mathrm{MS}) \times 100
$$

Taxa de Transpiração: A transpiração diária total da planta foi mensurada pela diferença de peso dos vasos. Inicialmente cada vaso foi inserido dentro de 
um saco plástico fixado com borracha no caule da planta, ficando apenas a parte aérea (folhas e caule) externa ao saco, em seguida, o conjunto vaso com planta e saco plástico foram pesados (massa 01), e 24 horas depois novamente pesados (massa 02). A transpiração total foi estimada pela diferença entre a massa 01 e massa 02.

Parâmetros de fluorescência: A análise de fluorescência da clorofila $a$ foi realizada utilizando fluorômetro portátil JUNIOR-PAM (Walz, Germany) no período escuro às 4:00 horas da manhã com emissão de um pulso de luz saturante de $0,3 \mathrm{~s}$, sob frequência de $0,6 \mathrm{KHz}$, aos 30 dias após a imposição do déficit hídrico. Os dados de fluorescência obtidos foram posteriormente computados utilizando-se o software WinControl-3.

Procedimentos estatísticos: As variáveis foram submetidas à análise de variância e teste de média de Newman-Keuls ao nível 5\% de probabilidade seguindo o delineamento inteiramente casualizado em esquema fatorial 2x5 (hormônio x níveis de irrigação) com cinco repetições. As variáveis foram submetidas à análise de regressão utilizando o software SigmaPlot10 (Sysstat, 2006). A análise multivariada foi feita por meio da regressão múltipla utilizando a seleção do modelo forward stepwise (Sokal e Rolf, 1995) e o software STATISTICA (Statsoft, 2007).

\section{Resultados}

A análise de variância para todas as variáveis analisadas encontra-se nas tabelas 1,2 e 3 . Para nenhuma das variáveis analisadas (Tabelas 1, 2 e 3) verificou-se diferenças significativas na interação entre hormônio e irrigação. Algumas variáveis obtiveram diferenças significativas para os fatores hormônio e irrigação isolados. $\mathrm{Na}$ Tabela 1, foi constatado efeito significativo dos diferentes níveis de irrigação para as variáveis altura de planta, diâmetro do caule (DCaule), área foliar (AF), transpiração $(E)$ e biomassa. Apenas as variáveis área foliar (AF), transpiração $(E)$ e biomassa obtiveram ajustes significativos a análise de regressão e, ajustaram ao modelo linear.

$\mathrm{Na}$ Tabela 2, as variavéis fluorecência máxima (Fm), rendimento quântico máximo do fotossistema II (Fv/Fm) e eficiência de captura de energia de excitação ( $\left.\mathrm{Fv}^{\prime} / \mathrm{Fm}^{\prime}\right)$ tiveram diferença significativa em relação a variação da irrigação na análise de variância. O hormônio interferiu significativamente nas variávies fluorescência inicial (F0), rendimento quântico máximo do fotossistema II ( $\mathrm{Fv} / \mathrm{Fm})$ e eficiência de captura de energia de excitação $\left(\mathrm{Fv}^{\prime} / \mathrm{Fm}^{\prime}\right)$. Para a análise de regressão as mesmas variáveis tiveram ajustes ao modelo linear, no entanto, somente a variável rendimento quântico máximo do fotossistema II (Fv/ Fm) foi significativa a $1 \%$ de probabilidade.

As variáveis razão massa foliar (RMF), razão massa radicular (RMR), carotenoides total (Car) e clorofila $a+b(\mathrm{Cl}$ Total $)$ obtiveram diferenças significativas em relação aos diferentes níveis de irrigação e, referente ao hormônio, apenas a variável ( $\mathrm{Cl}$ Total). Nenhuma das variáveis apresentadas na Tabela 3 ajustaram-se aos modelos de regressão linear ou quadrático.

Tabela 1. Análise de variância e teste de média para altura de planta, diâmetro de caule (DCaule), área foliar (AF), transpiração (E), teor relativo de água (TRA) e biomassa (Bio) de plantas de $E$. urocam submetidas a diferentes níveis de irrigação $(40,60,80,100$ e $120 \%$ da capacidade de retenção do substrato) e combinação de hormônios (cinetina, giberelina e brassinostenoides)

\begin{tabular}{|c|c|c|c|c|c|c|c|}
\hline \multicolumn{8}{|c|}{ Quadrados Médios } \\
\hline $\begin{array}{l}\text { Fonte de } \\
\text { Variação }\end{array}$ & GL & $\begin{array}{c}\text { Altura } \\
\text { (cm) }\end{array}$ & $\begin{array}{c}\text { DCaule } \\
(\mathrm{mm})\end{array}$ & $\begin{array}{c}\mathrm{AF} \\
(\mathrm{cm})\end{array}$ & $\begin{array}{c}E \\
(g)\end{array}$ & $\begin{array}{l}\text { TRA } \\
(\%)\end{array}$ & $\begin{array}{c}\text { Biomassa } \\
\text { (g) }\end{array}$ \\
\hline Hormônio (H) & 1 & $0,08^{\mathrm{ns}}$ & $0,205^{\mathrm{ns}}$ & $33627^{\mathrm{ns}}$ & $3024^{\mathrm{ns}}$ & $73,67^{\text {ns }}$ & $3,48^{\mathrm{ns}}$ \\
\hline Irrigação (I) & 4 & $257^{* *}$ & $1,901^{* *}$ & $536660^{* *}$ & $48564^{* *}$ & $13,82^{\text {ns }}$ & $32,57^{* *}$ \\
\hline $\mathrm{H}^{*} \mathrm{I}$ & 4 & $74,93^{\mathrm{ns}}$ & $0,207^{\mathrm{ns}}$ & $4954^{\mathrm{ns}}$ & $347^{\mathrm{ns}}$ & $10,07^{\mathrm{ns}}$ & $7,40^{\mathrm{ns}}$ \\
\hline Resíduo & 40 & 18,99 & 0,142 & 9596 & 1316,6 & 31,60 & 2,21 \\
\hline CV $(\%)$ & & 10,45 & 10,01 & 17,11 & 30,21 & 8,29 & 19,05 \\
\hline \multicolumn{8}{|c|}{ Médias } \\
\hline Com hormônio & & $41,68 \mathrm{a}$ & $3,78 \mathrm{a}$ & $598,3 \mathrm{a}$ & $127,8 \mathrm{a}$ & $66,61 \mathrm{a}$ & $8,07 \mathrm{a}$ \\
\hline Sem hormônio & & $41,76 \mathrm{a}$ & $3,74 \mathrm{a}$ & $545,9 \mathrm{a}$ & $112,0 \mathrm{a}$ & $69,07 \mathrm{a}$ & $7,54 \mathrm{a}$ \\
\hline \multicolumn{8}{|c|}{ Análise de regressão } \\
\hline Linear & & ns & ns & $* *$ & $* *$ & ns & $* *$ \\
\hline Quadrática & & $\mathrm{ns}$ & ns & $\mathrm{ns}$ & $\mathrm{ns}$ & ns & ns \\
\hline
\end{tabular}

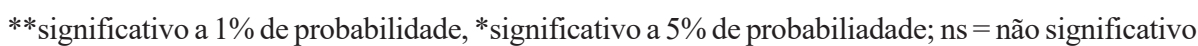
pelo teste F. Médias seguidas por uma mesma letra minúscula dentro da coluna não diferem entre si a $5 \%$ de probabilidade pelo teste de Newman-Keuls. 
Tabela 2. Análise de variância e teste de média para coeficiente de extinção fotoquímica (qP), fluorescência inicial $\left(\mathrm{F}_{0}\right)$, fluorescência máxima $(\mathrm{Fm})$, rendimento quântico máximo do fotossistema II (Fv/Fm), eficiência de captura de energia de excitação (Fv'/Fm') e energia absorvida não direcionada a fotoquímica nem dissipada por calor (Pe) de plantas de E. urocam submetidas a diferentes níveis de irrigação $(40,60,80,100$ e $120 \%$ da capacidade de retenção do substrato) e combinação de hormônios (cinetina, giberelina e brassinostenoides)

\begin{tabular}{|c|c|c|c|c|c|c|c|}
\hline \multicolumn{8}{|c|}{ Quadrados Médios } \\
\hline $\begin{array}{l}\text { Fonte de } \\
\text { Variação }\end{array}$ & GL & $\mathbf{q P}$ & $\mathbf{F}_{0}$ & Fm & $\mathbf{F v} / \mathbf{F m}$ & $\mathbf{F v} / \mathbf{F m}$ ' & $\mathbf{P e}$ \\
\hline Hormônio (H) & 1 & $0,0011^{\mathrm{ns}}$ & $110,9^{*}$ & $1,83^{\mathrm{ns}}$ & $0,01^{*}$ & $0,011^{*}$ & $0,0035^{\mathrm{ns}}$ \\
\hline Irrigação (I) & 4 & $0,0032^{\text {ns }}$ & $0,986^{\mathrm{ns}}$ & $761^{* *}$ & $0,004^{*}$ & $0,005^{*}$ & $0,0015^{\mathrm{ns}}$ \\
\hline $\mathrm{H}^{*} \mathrm{I}$ & 4 & $0,0043^{\mathrm{ns}}$ & $17,39^{\mathrm{ns}}$ & $359^{\mathrm{ns}}$ & $0,001^{\mathrm{ns}}$ & $0,001^{\mathrm{ns}}$ & $0,0026^{\mathrm{ns}}$ \\
\hline Resíduo & 40 & 0,0023 & 16,86 & 142 & 0,0016 & 0,0018 & 0,0016 \\
\hline CV $(\%)$ & & 6,84 & 20,24 & 13,63 & 5,27 & 5,63 & 17,84 \\
\hline \multicolumn{8}{|c|}{ Médias } \\
\hline Com hormônio & & $0,71 \mathrm{a}$ & $21,76 a$ & $87,72 \mathrm{a}$ & $0,76 b$ & $0,74 b$ & $0,21 \mathrm{a}$ \\
\hline Sem hormônio & & $0,70 \mathrm{a}$ & $18,75 b$ & $87,33 \mathrm{a}$ & $0,79 \mathrm{a}$ & $0,77 \mathrm{a}$ & $0,23 \mathrm{a}$ \\
\hline \multicolumn{8}{|c|}{ Análise de regressão } \\
\hline Linear & & $\mathrm{ns}$ & $\mathrm{ns}$ & $*$ & $* *$ & $*$ & ns \\
\hline Quadrática & & ns & $\mathrm{ns}$ & ns & ns & $\mathrm{ns}$ & ns \\
\hline
\end{tabular}

** significativo a $1 \%$ de probabilidade, ${ }^{*}$ significativo a $5 \%$ de probabiliadade; $n$ s = não significativo pelo teste F. Médias seguidas por uma mesma letra minúscula dentro da coluna não diferem entre si a $5 \%$ de probabilidade pelo teste de Newman-Keuls.

Tabela 3. Análise de variância e teste de média para razão massa caulina (RMC), razão massa foliar (RMF), razão massa radicular (RMR), carotenoides total (Car), coeficiente de extinção não fotoquímica (NPQ) e clorofila $a+b(\mathrm{Cl}$ Total) de plantas de $E$. urocam submetidas a diferentes níveis de irrigação $(40,60,80,100$ e $120 \%$ da capacidade de retenção do substrato) e combinação de hormônios (cinetina, giberelina e brassinostenoides)

\begin{tabular}{|c|c|c|c|c|c|c|c|}
\hline \multicolumn{8}{|c|}{ Quadrados Médios } \\
\hline $\begin{array}{l}\text { Fonte de } \\
\text { Variação }\end{array}$ & GL & RMC & RMF & RMR & $\begin{array}{c}\text { Car } \\
\left(\mathrm{cm}^{2}\right)\end{array}$ & NPQ & $\begin{array}{c}\text { Cl Total } \\
\text { (mg g MF) }\end{array}$ \\
\hline Hormônio (H) & 1 & $0,0018^{\mathrm{ns}}$ & $0,003^{\mathrm{ns}}$ & $0,009^{\mathrm{ns}}$ & $0,052^{\mathrm{ns}}$ & $0,00001^{\mathrm{ns}}$ & $3,964^{*}$ \\
\hline Irrigação (I) & 4 & $0,001^{\mathrm{ns}}$ & $0,014^{* *}$ & $0,021^{* *}$ & $0,14^{* *}$ & $0,0004^{\mathrm{ns}}$ & $3,320^{*}$ \\
\hline $\mathrm{H}^{*} \mathrm{I}$ & 4 & $0,0005^{\text {ns }}$ & $0,004^{\mathrm{ns}}$ & $0,002^{\mathrm{ns}}$ & $0,058^{\mathrm{ns}}$ & $0,001^{\mathrm{ns}}$ & $1,416^{\mathrm{ns}}$ \\
\hline Resíduo & 40 & 0,001 & 0,0019 & 0,0038 & 0,025 & 0,0009 & 0,964 \\
\hline CV $(\%)$ & & 17,83 & 9,65 & 17,39 & 18,22 & 83,38 & 20,21 \\
\hline \multicolumn{8}{|c|}{ Médias } \\
\hline Com hormônio & & $0,19 a$ & $0,46 \mathrm{a}$ & $0,34 \mathrm{a}$ & $0,84 \mathrm{a}$ & $0,036 \mathrm{a}$ & $4,57 b$ \\
\hline Sem hormônio & & $0,18 \mathrm{a}$ & $0,44 \mathrm{a}$ & $0,37 \mathrm{a}$ & $0,91 \mathrm{a}$ & $0,037 \mathrm{a}$ & $5,14 \mathrm{a}$ \\
\hline \multicolumn{8}{|c|}{ Análise de regressão } \\
\hline Linear & & ns & ns & $\mathrm{ns}$ & ns & ns & $\mathrm{ns}$ \\
\hline Quadrática & & ns & $\mathrm{ns}$ & $\mathrm{ns}$ & ns & $\mathrm{ns}$ & $\mathrm{ns}$ \\
\hline
\end{tabular}

** significativo a $1 \%$ de probabilidade, * significativo a $5 \%$ de probabiliadade; $n$ s = não significativo pelo teste F. Médias seguidas por uma mesma letra minúscula dentro da coluna não diferem entre si a $5 \%$ de probabilidade pelo teste de Newman-Keuls.

A análise de variância mostra que a aplicação dos hormônios combinados não apresentou efeito significativo no crescimento de plantas de E. urocam, no entanto, os diferentes volumes de água interferiram de forma linear na biomassa, área foliar, transpiração e máxima eficiência quântica do FSII (Figura 1).

A fluorescência inicial foi $14 \%$ superior nas plantas com hormônio em relação a testemunha enquanto a máxima eficiência quântica do FSII e eficiência de captura de radiação solar pelo FSII foram 4\% menores nas plantas com hormônio quando comparadas a testemunha sem hormônio (Tabela 2). As mesmas variáveis e a fluorescência máxima foram influenciadas significativamente de forma linear e crescente pelos diferentes volumes de água (Figura 1).

A análise de regressão múltipla avalia o efeito das variáveis analisadas no acúmulo de biomassa de plantas de E. urocam (Tabela 4). A área foliar, razão de massa foliar e clorofila total respondem por $85 \%$ da variação da biomassa, não obstante, a área foliar interferiu positivamente no acúmulo de biomassa, enquanto a razão de massa foliar e clorofilas totais interferiram negativamente.

\section{Discussão}

$\mathrm{O}$ uso de reguladores vegetais tem sido apontado como promissora prática de manejo de espécies 

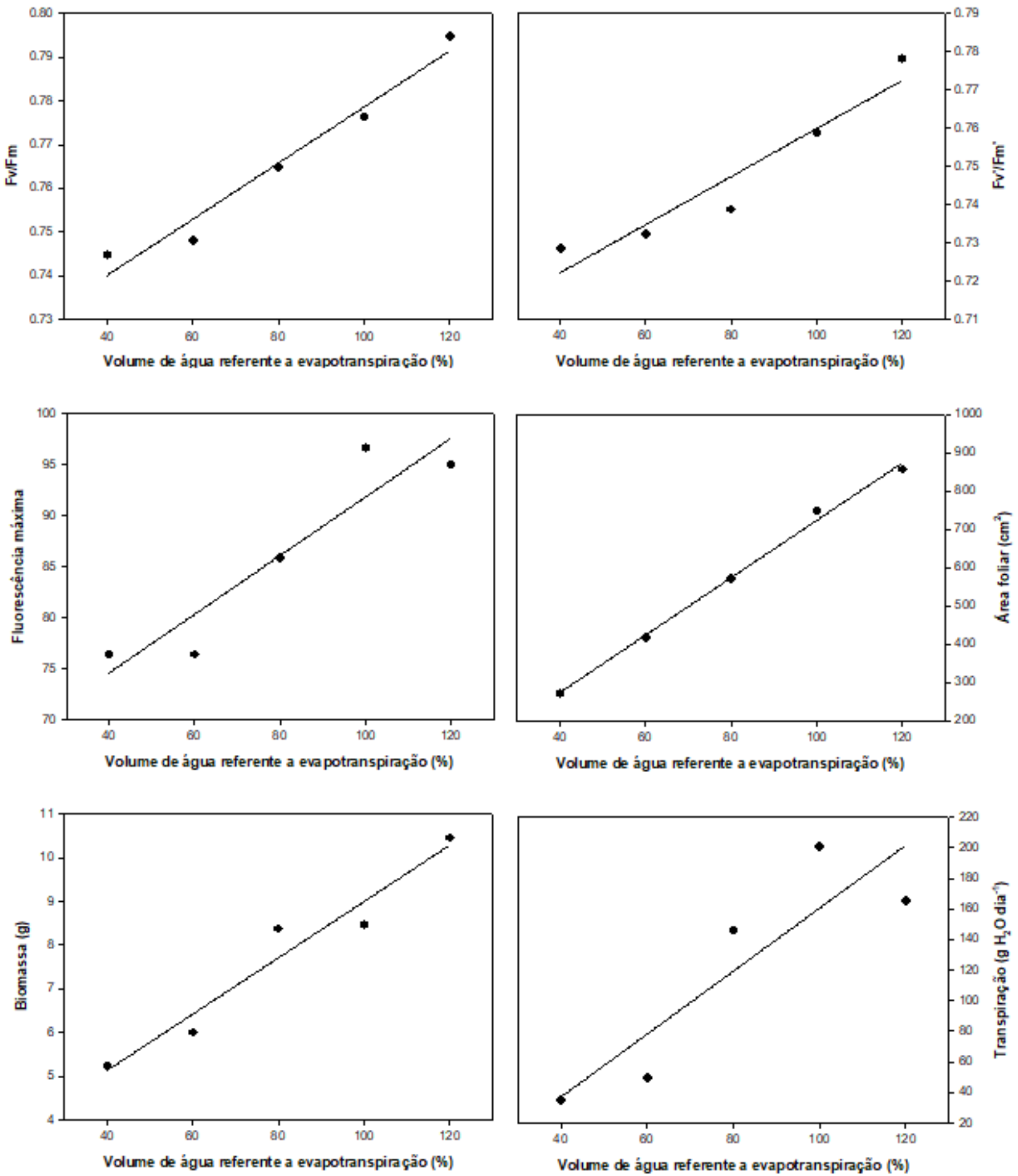

Figura 1. Equações de regressão para o máximo rendimento quântico do FSII: Fv/Fm=0,714 + 0,0006x, $\mathrm{R}^{2}=0,95^{* *}$, eficiência de captura da energia de excitação pelos centros de reação abertos do PSII: Fv'/Fm' $=0,697+0,0006 x$, $\mathrm{R}^{2}=0,91 * *$, fluorescência máxima: $\mathrm{Fm}=63,08+0,29, \mathrm{R}^{2}=0,87 *$, Área foliar $=-26,53+7,51 \mathrm{x}, \mathrm{R}^{2}=0,99^{* *}$, biomassa $=2,55+0,065 \mathrm{x}, \mathrm{R}^{2}=0,94 * *$ e transpiração $=-45,21$ $+2,06 \mathrm{x}, \mathrm{R}^{2}=0,80 *$ de plantas de E. urocam submetidas a diferentes níveis de irrigação (40, 60, 80, 100 e $120 \%$ da capacidade de retenção do substrato) e combinação de hormônios (cinetina, giberelina e brassinostenoides). 
Tabela 4. Modelo de regressão múltipla para avaliar o efeito das variáveis analisadas sobre a biomassa total de plantas de E. urocam submetidas a diferentes níveis de irrigação (40, 60, 80, 100 e $120 \%$ da capacidade de retenção do substrato) e combinação de hormônios (cinetina, giberelina e brassinostenoides)

\begin{tabular}{lcccccc}
\hline Biomassa & \multicolumn{2}{c}{$\mathbf{R}^{\mathbf{2}=\mathbf{0 , 8 5}}$} & \multicolumn{2}{c}{$\mathbf{F}(\mathbf{1 0 ; 3 9 )}) \mathbf{2 2 , 5 8}$} \\
& Beta & Std,Err, of Beta & B & Std,Err, of B & t (19) & p-level \\
\hline Intercept & & & 4,344 & 2,796 & 1,554 & 0,128 \\
Área foliar & 0,917 & 0,129 & 0,009 & 0,001 & 7,120 & 0,000 \\
RMF & $-0,462$ & 0,073 & $-18,981$ & 3,002 & $-6,323$ & 0,000 \\
Cl total & $-0,313$ & 0,114 & $-0,662$ & 0,241 & $-2,744$ & 0,009 \\
\hline
\end{tabular}

* Significativo a 1\%; RMF: Razão de massa foliar; $\mathrm{Cl}$ total: Clorofila total.

florestais no intuito de maximizar o crescimento inicial e facilitar o estabelecimento das plantas (Lopes et al., 2015). No presente estudo a utilização da mistura de reguladores vegetais (brassinosteroides, citocinina e giberelina) promoveu leves reduções na concentração de clorofilas e eficiências de captação de energia solar e eficiência quântica do FSII insuficientes para danos metabólicos e sintomas visuais de estresse. No entanto, esta ligeira diferença aliada à similaridade no crescimento de plantas submetidas aos hormônios e testemunha são suficientes para assegurar que os reguladores vegetais não proporcionaram variações morfofisiológicas significativas em plantas de E. urocam.

A sazonalidade das precipitações pluviométricas torna a escassez de água o mais frequente evento causador de estresse. A pressão do protoplasto na parede celular durante a turgidez e relaxamento enzimático da parede celular é condição sine qua non para o alongamento celular e, à medida que se aumentou o volume de água ocorreu incremento linear de área foliar, de forma que as plantas irrigadas com volume de água referente a $40 \%$ da capacidade de retenção do substrato apresentam área foliar $68 \%$ inferior às plantas irrigadas com $120 \%$. Os dados corroboram aos encontrados por Souza et al. (2015) avaliado plantas de eucalipto em estádio inicial de crescimento.

A maior área foliar representa elevado potencial de absorção de energia luminosa, alta fotossíntese e acúmulo de biomassa conforme registra a Tabela 4 . Os estômatos acoplam a transpiração a produção de biomassa, pois pelo mesmo poro de perda de água ocorre influxo de $\mathrm{CO}_{2}$, dessa forma, a maior taxa transpiratória em plantas irrigadas com volumes de água referentes a $120 \%$ da capacidade de retenção do substrato coincide com maior acúmulo de biomassa e área foliar, pois nesta condição de umidade a turgidez é suficiente para alongamento e incremento da área foliar e os estômatos abertos permitem a perda de água e o influxo de $\mathrm{CO}_{2}$. As variações na área foliar e biomassa também foram registrados em eucalipto por Yousaf et al. (2018).

As reduções na eficiência quântica do FSII (Fv/ Fm) e eficiência de captação de energia de excitação (Fv'/Fm') e fluorescência máxima em plantas sob déficit hídrico são indicativos de estresse pela redução na dissipação térmica dos centros de reação do FSII. Os resultados corroboram aos encontrados por Banks, (2018) que identificou variação da eficiência quântica do PS II (Fv/Fm) em plantas sob déficit hídrico.

A consonância entre a fluorescência, área foliar, biomassa e transpiração em plantas irrigadas com volumes de água variando de 40 a $120 \%$ da capacidade de retenção do substrato demonstra que o déficit hídrico exerceu efeito negativo na morfofisiologia do crescimento de E. urocam.

\section{Conclusão}

A deficiência hídrica reduz o desenvolvimento de plantas de E. urocam. Os reguladores vegetais utilizados em mudas de E. urocam não promove variações no desenvolvimento destas plantas quando submetidas a diferentes níveis de irrigação, por isso, não se justifica o uso em mudas desta espécie.

\section{Literatura Citada}

ALLEN, R. G. et al. 1998. Crop evapotranspiration: guidelines for computing crop water requirements. FAO. Irrigation and Dranaige Paper, 56. Rome, FAO. 300p. 
ANTWI-BOASIAKO, C.; ACHEAMPONG, B. B. 2016. Strengh properties and calorific values of sawdust-briquettes as Wood-residue energy generation source from tropical hardwoods of different densities. Biomass and Bioenergy 85:144-152.

ARCE, J. J. C. 2019. Background Analytical Study Forests, inclusive and sustainable economic growth and employment. Forests and SDG8. $58 \mathrm{p}$.

AWAN, F. K.; KHURSHID, Y.; MEHMOOD, A. 2017. Plant Growth Regulators and Their Role in Abiotic Stress Management. The International Journal of Innovative Research in Biosciences 1(1):9-22.

BANKS, J. M. 2018. Chlorophyll fluorescence as a tool to identify drought stress in Acer genotypes. Environmental and Experimental Botany 155:118-127.

BROOKER, M. I. H. 2000. A new classification of the genus Eucalyptus L'Her. (Myrtaceae). Australian Systematic Botany 13(1):79-148.

DRESSELHAUS, T.; HÜCKELHOVEN, R. 2018. Biotic and Abiotic Stress Responses in Crop Plants. Agronomy 8(11):1-6.

FOOD AND AGRICULTURE ORGANIZATION FAO. 2018. The state of the world's forests: Forest Pathways to sustainable development, Acesso em: 28/06/2019. Disponível em: http:// www.fao.org/3/19535EN/i9535en.pdf

FRANÇA, M. C. et al. 2019. Quality of the log and wood of Eucalyptus clones for use in lumber industry. Ciência da Madeira (Brasil) 10(1):8-17.

GLANTZ, M. 2019. Desertification: environmental degradation in and around arid lands. CRC Press. $366 \mathrm{p}$.

JONG, W. et al. 2018. Community forestry and the sustainable development goals: A two way street. Forests 9(6):01-18.
LOPES, V. A. et al. 2015. Initial growth of eucalyptus plants treated with gibberellin. African Journal of Agricultural Research 10(11):1251-1255.

MAGGIO, A. et al. 2018. It's Hard to Avoid Avoidance: Uncoupling the Evolutionary Connection between Plant Growth, Productivity and Stress "Tolerance". International Journal of Molecular Sciences 19(11):01-24.

MCMAHON, D. E.; JACKSON, R. B. 2019. Management intensification maintains wood production over multiple harvests in tropical Eucalyptus plantations. Ecological Applications 29(4):01-15.

SOKAL, R. R.; ROHLF, F. J. 1995. Biometry: the principals and practice of statistics in biological research. W. H. Freeman, New York.

SMITH, M. 1991. Report on the expert consultation on revision of FAO methodologies for crop water requiremebts. Rome FAO. $45 \mathrm{p}$.

STATSOFT, INC. 2007. Statistica (data analysis software system). Version 7. Acesso em 17 jun. 2019. Disponível em: http://www.statsoft.com/ Products/STATISTICA-Features

SYSSTAT SOFTWARE, INC - SSI. 2006. SigmaPlot for Windows. Version 10. Acesso em 17 jun. 2019. Disponível em: https://systatsoftware.com/ products/sigmaplot/

SOUZA, B. R. et al. 2015. Growth of Eucalyptus plants irrigated with saline water. African Journal of Agricultural Research 10(1):1091-1096.

WELLBURN, A. R. 1994. The spectral determination of chlorophylls a and $b$, as well as total carotenoids, using various solvents with spectrophotometers of different resolution. Journal of Plant Physiology 144(3):307-313.

YOUSAF, M. S. et al. 2018. Effect of Drought Stress on the Growth and Morphological Traits of Eucalyptus camaldulensis and Eucalyptus citriodora. PSM Biological Research 3(3):85-91. 